\title{
Daptomycin in the Clinical Setting: 8-Year Experience with Gram-positive Bacterial Infections from the EU-CORE ${ }^{S M}$ Registry
}

\author{
Armando Gonzalez-Ruiz • Panayiotis Gargalianos-Kakolyris • Artur Timerman • \\ Jayanta Sarma • Víctor José González Ramallo • Kamel Bouylout • \\ Uwe Trostmann · Rashidkhan Pathan · Kamal Hamed (D) \\ To view enhanced content go to www.advancesintherapy.com \\ Received: May 19, 2015 / Published online: June 25, 2015 \\ (C) The Author(s) 2015. This article is published with open access at Springerlink.com
}

\begin{abstract}
Introduction: The aim of this study was to evaluate the clinical outcomes and safety of daptomycin therapy in patients with serious Gram-positive infections.
\end{abstract}

Electronic supplementary material The online version of this article (doi:10.1007/s12325-015-0220-6) contains supplementary material, which is available to authorized users.

A. Gonzalez-Ruiz

Darent Valley Hospital, Dartford, UK

P. Gargalianos-Kakolyris

G. Gennimatas General Hospital of Athens, Athens, Greece

A. Timerman

Hospital Edmundo Vasconcelos, São Paulo, Brazil

J. Sarma

North Tyneside General Hospital, North Shields, UK

V. José González Ramallo

Gregorio Marañon General Hospital, Madrid, Spain

K. Bouylout · U. Trostmann

Novartis Pharma AG, Basel, Switzerland

R. Pathan

Novartis Healthcare Pvt. Ltd., Hyderabad, India

K. Hamed $(\bowtie)$

Novartis Pharmaceuticals Corporation, East

Hanover, NJ, USA

e-mail: kamal.hamed@novartis.com
Methods: Patients were enrolled in the European Cubicin ${ }^{\circledR}$ Outcomes Registry and Experience (EU-CORE $\left.{ }^{\mathrm{SM}}\right)$, a noninterventional, multicenter, observational registry. The real-world data were collected across 18 countries (Europe, Latin America, and Asia) for patients who had received at least one dose of daptomycin between January 2006 and April 2012. Two-year follow-up data were collected until 2014 for patients with endocarditis, intracardiac/intravascular device infection, osteomyelitis, or orthopedic device infection.

Results: A total of 6075 patients were enrolled. The most common primary infections were complicated skin and soft tissue infection (31.7\%) and bacteremia (20.7\%). Staphylococcus aureus was the most frequently reported pathogen (42.9\%; methicillin-resistant S. aureus [MRSA], 23.2\%), followed by Staphylococcus epidermidis and other coagulasenegative staphylococci (CoNS, 28.5\%). The most commonly prescribed dose of daptomycin was $6 \mathrm{mg} / \mathrm{kg} /$ day (43.6\%), and the median duration of therapy was 11 (range 1-300) days. Overall clinical success rate was $80.5 \%$, and was similar whether daptomycin 
was used as first-line (82.9\%) or second-line (79.2\%) therapy. Clinical success rates were high in patients with $S$. aureus (83.9\%; MRSA $83.0 \%$ ) and CoNS (including S. epidermidis, $82.5 \%$ infections. The majority of patients with endocarditis or intracardiac/intravascular device infection (86.7\%) or osteomyelitis/ orthopedic device infection (85.9\%) had a sustained response during the 2-year follow-up period. There were no new or unexpected safety findings.

Conclusion: Results from real-world clinical experience showed that daptomycin is a valuable therapeutic option in the management of various difficult-to-treat Grampositive infections.

Funding: This study was funded by Novartis Pharma AG.

Keywords: Clinical response; Daptomycin; Gram-positive infections; Registry; Safety; Staphylococcus aureus

\section{INTRODUCTION}

Infections caused by Gram-positive pathogens are frequently encountered in the healthcare setting and are associated with high morbidity and mortality [1]. Complicated skin and soft tissue infections (cSSTIs) caused by Staphylococcus aureus are among the most common bacterial infections, accounting for approximately $25 \%$ of all infections in clinical practice $[1,2]$. Other Gram-positive bacterial infections include endocarditis, bacteremia, osteomyelitis, and foreign body or prosthetic device-related infections which are known to be difficult to treat.

Staphylococcus aureus is considered to be the most important cause of healthcare and community-associated infections worldwide [3,
4]. The European Union/European Economic Area population-weighted mean percentage of methicillin-resistant $S$. aureus (MRSA) infection was $18 \%$, whereas 7 out of the 30 reporting countries had a prevalence of MRSA infections above 25\% [5]. Among the available conventional therapies, vancomycin has been considered to be the main treatment option for MRSA infections [6]. Nonetheless, development of resistance and changes in MRSA susceptibility to vancomycin with increasing minimum inhibitory concentrations (MICs) have been observed [7]. Evidence supports the association between increased vancomycin MIC and worse patient outcome, with higher mortality in patients with bacteremia [8, 9]. In addition, slower clinical response and increased relapse rate have been associated with infections caused by MRSA with a high vancomycin MIC [10]. Although vancomycin has been used as an alternative treatment for enterococci, the emergence of vancomycin-resistant enterococci (VRE) as leading cause of several nosocomial infections is a serious concern. Several other limitations of vancomycin use have been recognized such as poor tissue penetration, dosing in patients with renal failure and synergistic toxicity with concomitant aminoglycoside administration $[6,11]$.

Resistance to currently available antibiotics is an alarming challenge in clinical settings [1214]. As empirical treatment is often initiated where appropriate before identification of the infecting pathogen, first-line use of an antibiotic effective against resistant pathogens including MRSA is therefore preferable. The increased frequency of Gram-positive infections and the rise in resistance to commonly used antibiotics have led to the need for novel antibiotics $[6,14]$.

Daptomycin is a cyclic lipopeptide with rapid bactericidal activity against a wide range of 
Gram-positive bacteria including MRSA and $S$. aureus with increased vancomycin MIC values. It is approved for the treatment of cSSTIs ( $4 \mathrm{mg} / \mathrm{kg} /$ day), right-sided infective endocarditis (RIE) due to $S$. aureus and bacteremia associated with cSSTIs or RIE (6 mg/kg/day) [15]. It offers rapid recovery from infections, which reduces the risk of resistance development, and may shorten hospitalization and overall treatment costs [16, 17]. Further, as compared to vancomycin, daptomycin has a favorable renal safety profile with prolonged use $[18,19]$.

The clinical experience with daptomycin since its approval in Europe, Latin America, and Asia has been captured by the European Cubicin ${ }^{\circledR}$ Outcomes Registry and Experience $\left(\mathrm{EU}-\mathrm{CORE}^{\mathrm{SM}}\right)$. EU-CORE, a retrospective, multicenter, and non-interventional study, was designed to collect real-world data of daptomycin treatment for Gram-positive infections. Interim results of EU-CORE were also published by Gonzalez-Ruiz and colleagues [20]. The objectives of this observational registry were to assess the clinical outcomes and safety of daptomycin in a large multicenter cohort of patients in clinical practice to reflect the real-world experience with daptomycin use.

\section{METHODS}

\section{Patients and Data Collection}

This analysis included data from patients across 18 countries in Europe (12), Latin America (5), and Asia (1) who had received at least one dose of daptomycin between January 2006 and April 2012 for the treatment of serious Gram-positive bacterial infections. All patients were followed up for a period of 30 days post-treatment, and patients with endocarditis, intracardiac/ intravascular device infection, osteomyelitis, or orthopedic device infection were followed up for 2 years until 2014. The protocol was approved by the health authority and the Institutional Review Board (IRB) or Ethics Committee (EC) in each country and written informed consent was obtained according to the requirements of the IRB or EC and/or the local data privacy regulations. Patients were included in the study if they were treated with at least one dose of daptomycin and if all mandatory information was available in the hospital files. Patients who received daptomycin as part of a controlled clinical trial were not eligible for inclusion in the study.

Investigators collected demographic, antibiotic, clinical, and microbiologic data from medical records at each site, as previously reported [20].

\section{Clinical Outcomes}

Clinical outcomes were assessed by the investigators at the end of daptomycin therapy according to protocol-defined criteria: cured, clinical signs and symptoms resolved, no additional antibiotic therapy was necessary, or infection cleared with a negative culture reported; improved, partial resolution of clinical signs and symptoms and/or additional antibiotic therapy was warranted; failed, inadequate response to daptomycin therapy, worsening or new/recurrent signs and symptoms, need for a change in antibiotic therapy, or a positive culture reported at the end of the therapy; and non-evaluable, unable to determine response due to insufficient information. Clinical success was defined as outcome of cured or improved. Time to improvement was recorded. The reasons for stopping daptomycin therapy and other antibiotics prescribed following daptomycin were also collected. 
The duration of treatment was evaluated as the number of inpatient and outpatient days during which the patient received daptomycin therapy, even if they were non-consecutive. There were no restrictions on concomitant treatment. Data on prior and concomitant antibiotic therapy were collected.

\section{Safety}

Adverse events (AEs) and serious AEs (SAEs) reported during daptomycin treatment and after 30 days from the end of daptomycin therapy were recorded, regardless of the study drug relationship. Microbiologic data included the culture results obtained before or shortly after the initiation of daptomycin therapy.

\section{Statistical Analysis}

Statistical analysis was performed using SAS version 9.3 (SAS Institute Inc., Cary, NC, USA). Due to the nature of the trial, inferential analyses were not conducted and no formal statistical methodology other than simple descriptive statistics was used. All analyses were considered to be explanatory.

Numerical variables were summarized as arithmetic mean, standard deviation, median, minimum, first quartile, third quartile, and maximum for continuous variables. Categorical variables were summarized by absolute and relative frequencies.

\section{RESULTS}

\section{Patient Demographics and Clinical Characteristics}

Overall, 6075 patients were included in the safety population; of these, 81 were pediatric patients. The patient demographic and clinical characteristics are described in Table 1 . The median age was 62.0 years. A total of 2777 $(45.7 \%)$ patients were aged $\geq 65$ years, including 1284 (21.1\%) aged $\geq 75$ years. Comorbidity was frequent as would be expected in seriously

Table 1 Patient demographic and baseline clinical characteristics (safety population)

\begin{tabular}{|c|c|c|}
\hline \multirow[t]{2}{*}{ Characteristics } & \multicolumn{2}{|c|}{$\begin{array}{l}N=6075 \\
n(\%)\end{array}$} \\
\hline & & \\
\hline Male & 3788 & $(62.4)$ \\
\hline Female & 2287 & $(37.6)$ \\
\hline Age (years), median (range) & 62 & $(1-103)$ \\
\hline$<18$ & 81 & $(1.3)$ \\
\hline$\geq 18$ to $<65$ & 3212 & $(52.9)$ \\
\hline$\geq 65$ (including $\geq 75$ ) & 2777 & $(45.7)$ \\
\hline$\geq 75$ & 1284 & $(21.1)$ \\
\hline Race, Caucasian & 5224 & $(86.0)$ \\
\hline Body weight (kg), median (range) & 75.0 & $(6-200)$ \\
\hline $\begin{array}{l}\text { Renal impairment at the initiation of } \\
\text { daptomycin therapy } \\
(\mathrm{CrCl}<30 \mathrm{~mL} / \mathrm{min})\end{array}$ & 787 & $(13.0)$ \\
\hline $\begin{array}{l}\text { Patients on renal replacement therapy } \\
\text { at the initiation of daptomycin } \\
\text { therapy }\end{array}$ & 552 & $(9.1)$ \\
\hline \multicolumn{3}{|l|}{ Significant underlying disease $(>10 \%)$} \\
\hline Cardiovascular & 3322 & $(54.7)$ \\
\hline Diabetes mellitus & 1598 & $(26.3)$ \\
\hline Renal & 974 & $(16.0)$ \\
\hline Malignancy & 925 & $(15.2)$ \\
\hline Pulmonary & 804 & $(13.2)$ \\
\hline Gastrointestinal & 719 & $(11.8)$ \\
\hline
\end{tabular}

Data are presented as $n(\%)$ unless otherwise indicated $\mathrm{CrCl}$ creatinine clearance 
Table 2 Type of primary infection (safety population)

\begin{tabular}{lll}
\hline Infection type & \multicolumn{2}{l}{$\boldsymbol{N = 6 0 7 5}$} \\
& $\boldsymbol{n}(\%)$ & \\
\hline Complicated skin and soft tissue infections & 1927 & $(31.7)$ \\
Bacteremia & 1255 & $(20.7)$ \\
$\begin{array}{l}\text { Uncomplicated skin and soft tissue } \\
\text { infections }\end{array}$ & 646 & $(10.6)$ \\
$\begin{array}{l}\text { Endocarditis } \\
\text { Foreign body/prosthetic infection }\end{array}$ & 610 & $(10.0)$ \\
$\begin{array}{l}\text { Osteomyelitis (non-prosthetic and } \\
\text { prosthetic device related) }\end{array}$ & 595 & $(9.8)$ \\
Other & 432 & $(7.1)$ \\
\hline
\end{tabular}

${ }^{a}$ Includes septic arthritis, urinary tract infection/ pyelonephritis, necrotizing infections, necrotizing fasciitis, surgical/non-surgical antibiotic prophylaxis, neutropenic fever, CNS infection, metastatic abscess, and unknown or not otherwise specified infections

unwell and older patients, and $87.9 \%$ of patients had significant underlying disease. The most common underlying conditions were cardiovascular disease (54.7\%) and diabetes mellitus $(26.3 \%)$. The most frequently reported primary infections were cSSTIs (31.7\%) and bacteremia (20.7\%), followed by uncomplicated skin and soft tissue infections (uSSTIs, 10.6\%) and endocarditis (10.0\%;

Table 2).

\section{Microbiology}

Samples for cultures were obtained from 5038 (82.9\%) patients, of whom 3910 (77.6\%) had positive cultures. $S$. aureus was the most commonly isolated primary pathogen $(42.9 \%)$ in patients with positive cultures and MRSA was identified in $23.2 \%$ of patients (Table 3 ). Staphylococcus epidermidis was the most common coagulase-negative staphylococcal (CoNS) pathogen (16.5\%; Table 3).
Table 3 Primary pathogens in patients with positive cultures

\begin{tabular}{lrll}
\hline Primary pathogen isolated & \multicolumn{2}{c}{$\begin{array}{l}N=3910 \\
n(\%)\end{array}$} \\
\hline Staphylococcus aureus & 1676 & $(42.9)$ \\
Methicillin-resistant & 906 & $(23.2)$ \\
Methicillin-susceptible & 643 & $(16.4)$ \\
Methicillin susceptibility unknown & 127 & $(3.2)$ \\
Coagulase-negative staphylococci & 1116 & $(28.5)$ \\
Staphylococcus epidermidis & 644 & $(16.5)$ \\
Other & 472 & $(12.1)$ \\
Staphylococcus species coagulase not & 41 & $(1.0)$ \\
specified & & \\
Streptococcus agalactiae or group B & 30 & $(0.8)$ \\
streptococci & & \\
Streptococcus pyogenes or group A & 27 & $(0.7)$ \\
streptococci & & \\
Viridans streptococci group & 57 & $(1.5)$ \\
Other Streptococcus species & 64 & $(1.6)$ \\
Enterococcus faecalis & 236 & $(6.0)$ \\
Enterococcus faecium & 184 & $(4.7)$ \\
Vancomycin-resistant & 64 & $(1.6)$ \\
$\quad$ E. faecalis or E. faecium) & & \\
Other Enterococcus species & 52 & $(1.3)$ \\
Gram-negative bacilli & 231 & $(5.9)$ \\
Other & 196 & $(5.0)$ \\
\hline & & & \\
\hline & & &
\end{tabular}

${ }^{a}$ Includes Clostridium perfringens, Clostridium species, Corynebacterium species, Peptostreptococcus species, Gramnegative cocci, Gram-positive bacilli, Gram-positive cocci, viruses, fungi/yeast, and invalid/ambiguous pathogen code

\section{Previous and Concomitant Antibiotic Therapies}

Daptomycin was administered empirically in 3438 (56.6\%) patients. A total of 3966 (65.3\%) patients received antibiotics prior to daptomycin therapy. The most commonly 
used prior antibiotics were penicillins (25.4\%) and glycopeptides (24.4\%). Vancomycin was administered as prior therapy in 1052 (17.3\%) patients. The main reasons for the switch to daptomycin were failure of the previous antibiotic and narrowing of antibiotic therapy. A majority of patients $(n=3934,64.8 \%)$ received concomitant antibiotics with daptomycin as inpatient therapy, most commonly carbapenems (23.2\%) and penicillins (13.9\%).

\section{Daptomycin Prescribing Patterns}

The most frequently prescribed dose of daptomycin was $6 \mathrm{mg} / \mathrm{kg} /$ day in 2649 (43.6\%) patients; 1554 (25.6\%) patients received $4 \mathrm{mg} / \mathrm{kg} /$ day. A dose of $4 \mathrm{mg} / \mathrm{kg} /$ day was most frequently used in skin infections and $6 \mathrm{mg} / \mathrm{kg} /$ day dose was used for other infections such as bacteremia, endocarditis, and osteomyelitis. A total of 1097 (18.1\%) patients received doses $>6 \mathrm{mg} / \mathrm{kg} /$ day and $645(10.6 \%)$ patients received doses $\geq 8 \mathrm{mg} / \mathrm{kg} / \mathrm{day}$. Daptomycin treatment was received by 5879 (96.8\%) patients as inpatient therapy and 709 (11.7\%) patients as outpatient therapy. The median duration of daptomycin therapy was 11 (range 1-300) days; 10 (range 1-246) days for inpatients $(n=5879)$ and 14 (range 1-290) days for outpatients $(n=709)$. A total of 1892 $(31.1 \%)$ patients received daptomycin as firstline therapy and 3965 (65.3\%) patients as second-line therapy.

\section{Clinical Outcomes}

The overall clinical success rate achieved with daptomycin treatment was $80.5 \%$, and the rates were similar when daptomycin was used as firstline $(82.9 \%)$ or second-line therapy $(79.2 \%)$. The clinical success rates across different infections were similar (Fig. 1). Success rate by infection type independent of the treatment

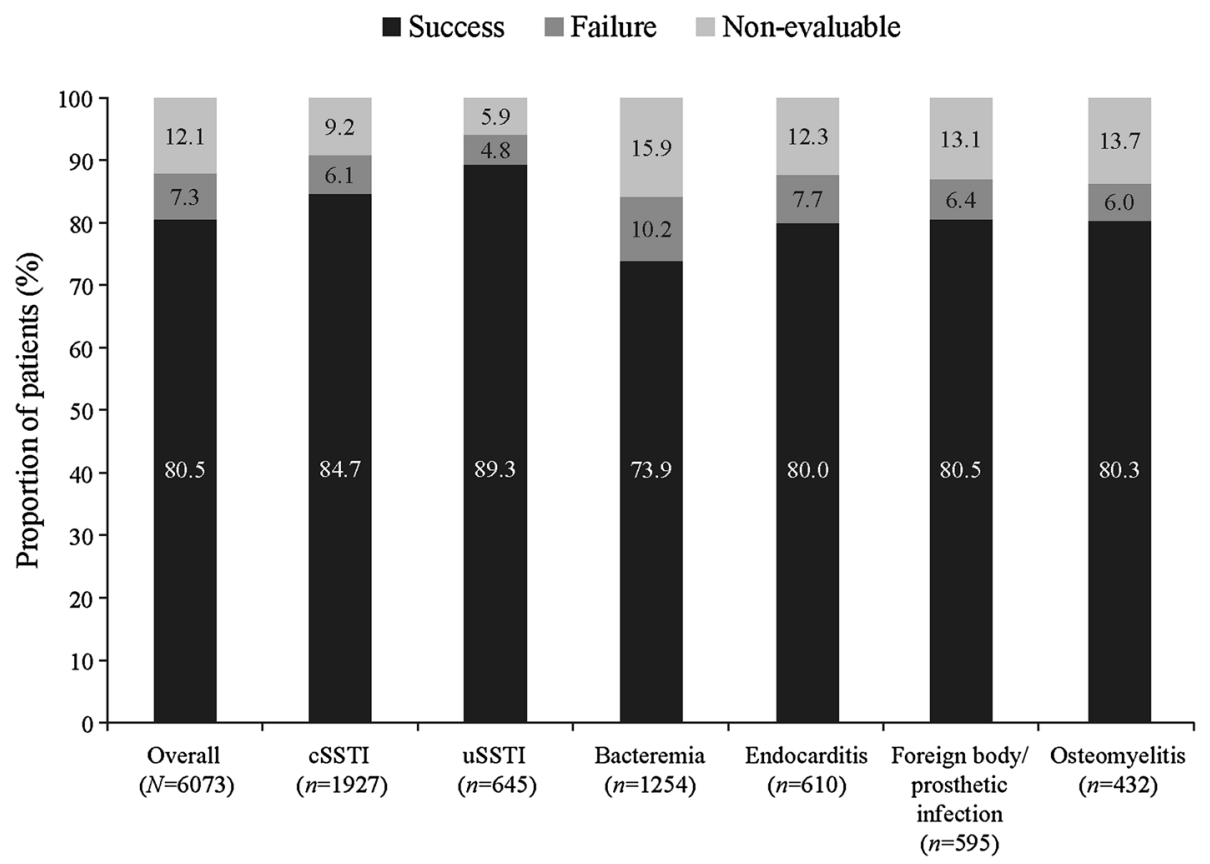

Fig. 1 Clinical outcome by primary infection. cSSTI complicated skin and soft tissue infection, $u S S T I$ uncomplicated skin and soft tissue infection 
dose ranged between $73.9 \%$ for bacteremia and $89.3 \%$ for uSSTIs. Low failure rates were reported for various primary infections (Fig. 1). As shown in Fig. 2, the clinical success rates by infecting pathogen were high for $S$. aureus. The clinical success rates showed an increasing trend with increasing daptomycin dose for endocarditis and foreign body/prosthetic infections (Fig. 3). Higher clinical success rates were also observed with increased duration of daptomycin therapy (Fig.4). The overall median time to improvement from initiation of daptomycin treatment was 4 (range 0-400) days.

The majority of patients (59.4\%) completed daptomycin therapy without further antibiotic treatment and $22.0 \%$ switched to another antibiotic after the end of the daptomycin therapy following improvement (e.g., stepdown to oral antibiotic therapy) or treatment failure. Within the subset of patients who received concomitant antibiotic therapy, the

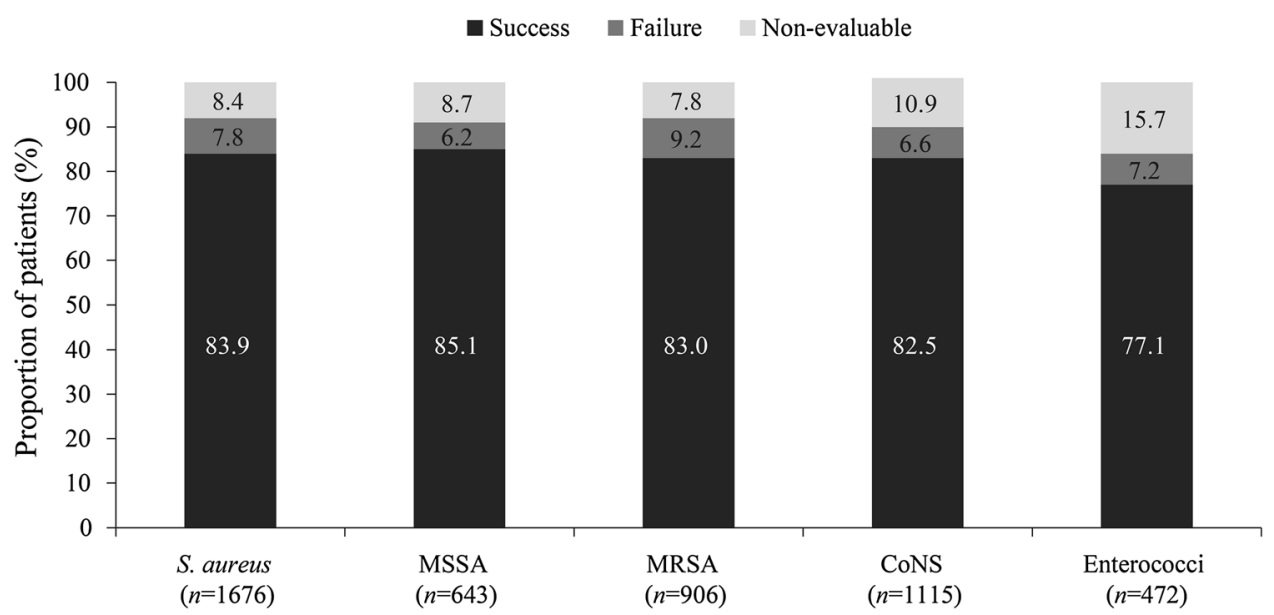

Fig. 2 Clinical outcome by infecting pathogen. Enterococci include Enterococcus faecalis, Enterococcus faecium and Enterococcus species. CoNS coagulase-negative

staphylococci, MRSA methicillin-resistant Staphylococcus aureus, MSSA methicillin-susceptible Staphylococcus aureus

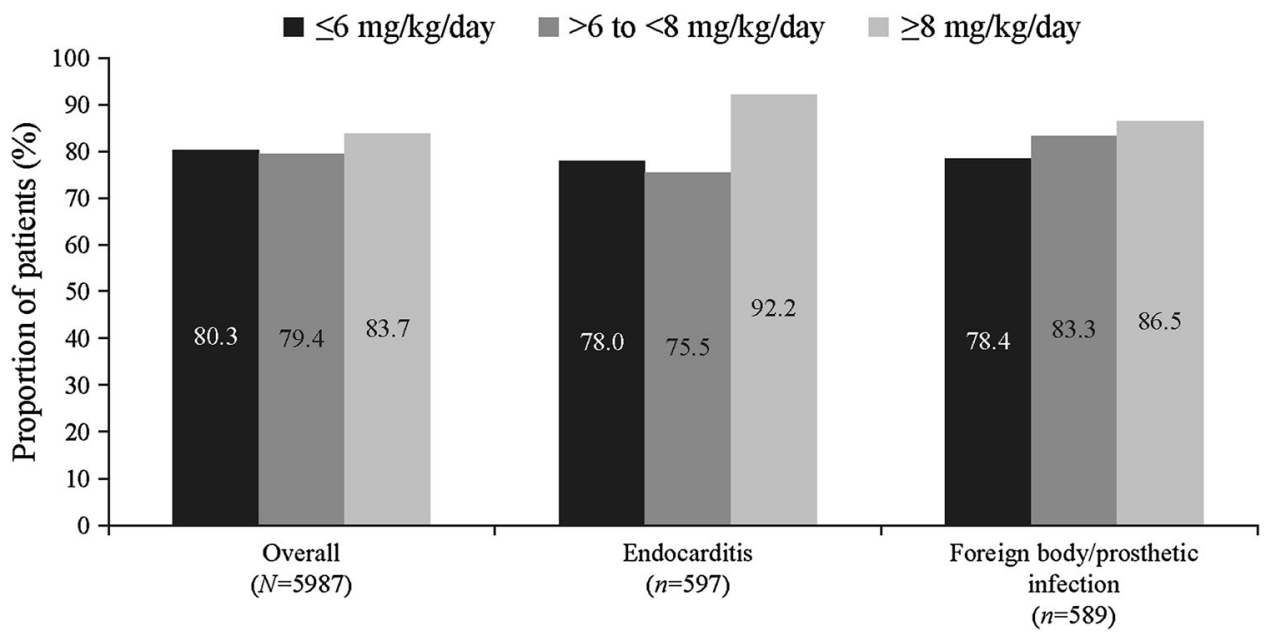

Fig. 3 Clinical success rates for endocarditis and foreign body/prosthetic infections by daptomycin dose. Patients with unknown dose information ( 86 overall, 13 endocarditis and 6 foreign body/prosthetic infection) were not included 


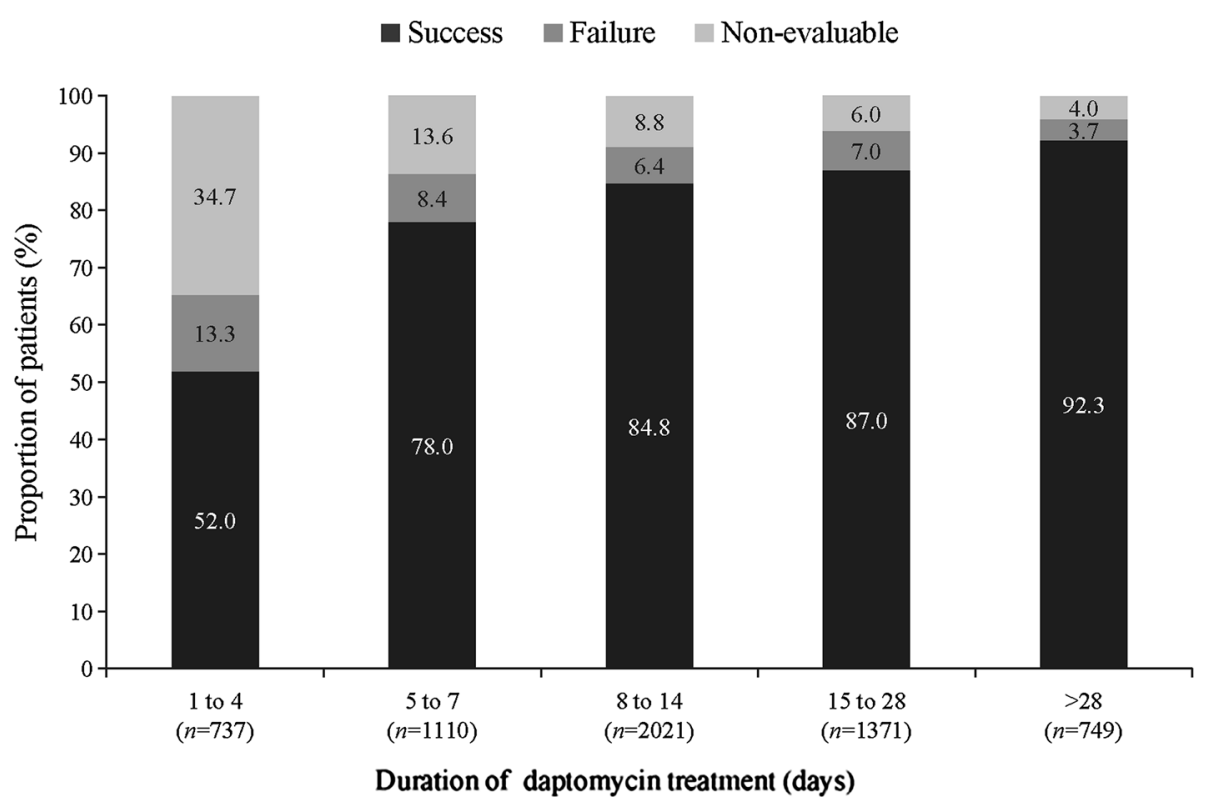

Fig. 4 Overall clinical outcome by duration of daptomycin therapy

clinical success rate was $77.7 \%$ as compared to $86.7 \%$ in patients who received no concomitant antibiotic therapy. The majority of patients with endocarditis or intracardiac/intravascular device infection $(86.7 \%)$ or osteomyelitis/orthopedic device infection (85.9\%) had a sustained response during the 2-year follow-up period.

\section{Safety}

Safety data from 6075 patients were included in this analysis. A total of 866 (14.3\%) patients reported at least one $\mathrm{AE}$ and 581 (9.6\%) patients reported SAEs (Table 4). AEs and SAEs possibly related to daptomycin therapy were reported in $193(3.2 \%)$ and $49(0.8 \%)$ patients, respectively. The most common AEs, possibly related to daptomycin, by system organ class were investigations $(n=62,1.0 \%), \quad$ skin and subcutaneous tissue disorders $(n=33,0.5 \%)$, and general disorders and administration site conditions ( $n=21,0.3 \%)$. The most frequently reported SAEs, possibly related to daptomycin, by system organ class were investigations
Table 4 Safety of daptomycin treatment in overall EU-CORE population

\begin{tabular}{lccc}
\hline Safety parameters & \multicolumn{2}{l}{$\begin{array}{l}N=6075 \\
n(\%)\end{array}$} \\
\hline Any AE & 866 & $(14.3)$ \\
AE possibly related to daptomycin & 193 & $(3.2)$ \\
AE leading to study drug discontinuation & 252 & $(4.1)$ \\
Any SAE & 581 & $(9.6)$ \\
SAE possibly related to daptomycin & 49 & $(0.8)$ \\
SAE leading to study drug discontinuation & 175 & $(2.9)$ \\
AEs occurring in $>1 \%$ patients, $n(\%)$ & & \\
Multi-organ failure & 86 & $(1.4)$ \\
Blood CPK increased & 76 & $(1.3)$ \\
Septic shock & 75 & $(1.2)$ \\
Sepsis & 73 & $(1.2)$ \\
\hline
\end{tabular}

$A E$ adverse event, $C P K$ creatine phosphokinase, $S A E$ serious $\mathrm{AE}$

$(n=12, \quad 0.2 \%)$, renal and urinary disorders $(n=11,0.2 \%)$, and general disorders and administration site conditions $(n=6,0.1 \%)$. Infections and infestations $(n=78,1.3 \%)$, 


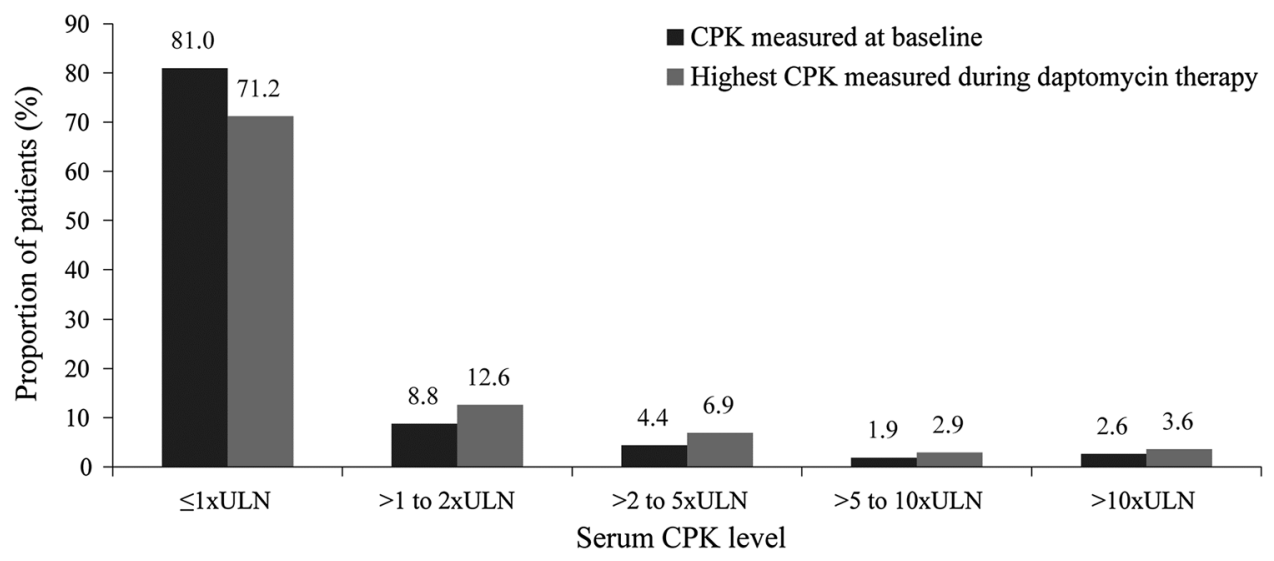

Fig. 5 Baseline and peak serum CPK concentrations. Values were missing for 49 patients at baseline and for 100 patients during the daptomycin therapy. $C P K$ creatine phosphokinase, $U L N$ upper limit of normal

general disorders and administration site conditions $(n=41,0.7 \%)$, and investigations $(n=38,0.6 \%)$ were the most frequently reported AEs by system organ class, regardless of relationship to daptomycin, which led to discontinuation of study medication.

An increased blood creatine phosphokinase (CPK) level was reported as an $\mathrm{AE}$ in 76 (1.3\%) patients, including 11 reported as SAEs. Musculoskeletal and connective tissue disorders were reported as AEs in $25(0.4 \%)$ patients. Out of these 25 patients, 12 had CPK levels increased to $>10 \times$ upper limit of normal (ULN) from baseline and 13 reported musculoskeletal and connective tissue disorders as SAEs of which 5 were reported as possibly related to daptomycin. Severe skeletal muscle toxicity was reported in $9(0.1 \%)$ patients. Rhabdomyolysis was reported as a SAE in $6(0.1 \%)$ patients, with 5 of the 6 SAEs considered as possibly related to daptomycin treatment by the investigator.

Serum CPK levels were measured at baseline in 3511 patients and the majority $(n=2843$, 81.0\%) had normal CPK values. At baseline, 92 (2.6\%) patients had CPK levels $>10 \times$ ULN. Fiftytwo patients $(40,6$, and 6 patients treated with $\leq 6$, $>6$ to $<8$, and $\geq 8 \mathrm{mg} / \mathrm{kg} /$ day, respectively) experienced a shift of CPK elevation from $\leq 10 \times$ ULN at baseline to $>10 \times$ ULN and 49 patients had a missing assessment. Peak serum CPK concentrations were in the range of the ULN throughout the analysis period in most patients (Fig. 5).

The total number of reported deaths during the study was $408(6.7 \%)$. Five $(0.1 \%)$ deaths were reported to be related to the study medication. The main causes of death were infections and infestations (3.4\%), general disorders and administration site conditions $(1.8 \%)$, multi-organ failure $(1.4 \%)$, sepsis $(1.3 \%)$, septic shock (1.2\%), and cardiac disorders (1.1\%).

\section{DISCUSSION}

The data from the EU-CORE registry illustrate 8 years of clinical experience of real-world usage of daptomycin against a variety of Grampositive infections including drug-resistant pathogens (MRSA, CoNS, and VRE) in patients with multiple co-morbidities. Overall, daptomycin used either as first- or second-line therapy was associated with high clinical success rates. The data demonstrated a favorable effectiveness and safety profile of 
daptomycin in the real-world setting, expanding on the results from randomized clinical trials and analyses of interim data from EU-CORE [20-22]. Patients from many sites across the 18 countries in Europe, Latin America, and Asia were enrolled in EU-CORE, allowing for the inclusion of a wide spectrum of infections and microbiologic data to be studied.

In this registry, although cSSTIs and bacteremia were the most common infections, daptomycin was also used to treat severe and deep-seated infections such as osteomyelitis and foreign body/prosthetic infections. These infections are of increasing clinical importance, where long-term treatment options are limited [23, 24]. Daptomycin retains advantage in this context because of its suggested activity in biofilms $[25,26]$. The clinical success rates for different primary infections were high. Daptomycin was also demonstrated to be effective against VRE infections that are generally challenging to treat. In addition, the rapid bactericidal activity of daptomycin is expected to reduce the opportunity for development of potential resistance [18, 21, 27, 28].

Data from previous studies suggest that daptomycin is mostly used as second-line therapy; however, in approximately $10-40 \%$ of patients it is used as first-line therapy, based on type of infection [29]. In this study, daptomycin showed favorable effectiveness whether used as first-line (83.0\%) or second-line (79.2\%) therapy with or without concomitant antibiotics. Daptomycin has an important role as first-line therapy for Gram-positive infections in terms of both effectiveness and cost considerations [21, 30]. Additionally, the present study demonstrated similar effectiveness with daptomycin treatment against both MRSA and methicillin-susceptible $S$. aureus infections, thus supporting its use as an empirical therapy for
S. aureus infections. This observation is in line with published guidelines that recommend daptomycin as the first alternative to vancomycin [31, 32].

The recommended first-line therapies for treating MRSA infections are vancomycin and linezolid; however, these are associated with various safety concerns, particularly in longterm use. Nephrotoxicity and ototoxicity are the known major adverse reactions related to vancomycin use. The monitoring of trough concentrations to prevent nephrotoxicity is recommended (i.e., sustained troughs of $15-20 \mathrm{~g} / \mathrm{mL}$ ), especially in patients with unstable renal function or therapy for longer than 3-5 days [33, 34]. On the other hand, linezolid therapy has been reported to be associated with myelosuppression, peripheral and optic neuropathy, and lactic acidosis especially with prolonged use [35]. Further, linezolid-associated serotonergic and adrenergic drug interactions can lead to severe AEs such as hypertensive episodes [36]. The rates of AEs reported for daptomycin in this retrospective observational study were low, although these may not be compared to $\mathrm{AE}$ reporting in a controlled clinical trial [23].

Most patients in this study received doses up to $6 \mathrm{mg} / \mathrm{kg} /$ day of daptomycin and $18 \%$ received doses $>6 \mathrm{mg} / \mathrm{kg} /$ day. No new or unexpected safety findings were reported even when patients received doses $>6 \mathrm{mg} / \mathrm{kg} /$ day (including doses $>10 \mathrm{mg} / \mathrm{kg} /$ day). On the basis of the linear pharmacokinetic profile and concentration-dependent activity of daptomycin, doses $>6 \mathrm{mg} / \mathrm{kg} /$ day are increasingly utilized [35, 37, 38]. Higher doses are also recommended for endocarditis and osteomyelitis by the Infectious Diseases Society of America guidelines [31, 39]. Furthermore, toxicity may be a concern while increasing the dose of daptomycin and previous 
reports showed that high-dose daptomycin ( $>6 \mathrm{mg} / \mathrm{kg} /$ day) may cause elevations in CPK levels at an incidence rate of 2.5-8.3\% [27, 4047]. However, CPK elevation during daptomycin therapy is not always associated with adverse musculoskeletal effects [40, 48]. In this study, a small proportion of patients experienced serum CPK elevation (1.7\%) and severe skeletal muscle toxicity $(0.1 \%)$.

Although rare cases of eosinophilic pneumonia were reported as AEs related to daptomycin (3 patients), patients recovered upon discontinuation of daptomycin therapy. Altogether, these outcomes reaffirm the safety profile of daptomycin treatment.

The inclusive nature of the registry was a strength; however, there are some inherent limitations such as the non-randomized, nonblinded design, and the patient outcomes that were solely determined by the treating physician. Despite these limitations, this registry reflects real-world clinical settings and permits the inclusion of diverse infections treated with concomitant antibiotics.

\section{CONCLUSIONS}

These results from 8 years of clinical experience complement the data from randomized clinical studies and show that daptomycin is a valuable treatment option in the management of various Gram-positive infections including those which are difficult to treat. However, further data explorations are required to examine infection- and population-specific outcomes within the EU-CORE registry in detail.

\section{ACKNOWLEDGMENTS}

Sponsorship, article processing charges, and the open access charge for this study were funded by Novartis Pharma AG. All named authors meet the International Committee of Medical Journal Editors (ICMJE) criteria for authorship of this manuscript, take responsibility for the integrity of the work as a whole, and have given final approval to the version to be published. These data were presented in part at the 25th European Congress of Clinical Microbiology and Infectious Diseases, Copenhagen, Denmark, 2015 (abstract 1233). Medical writing support was provided by Anupama Tamta (Novartis Healthcare Pvt. Ltd., Hyderabad, India) and Farid Khalfi (Novartis Ireland Ltd., Dublin, Ireland). Funding for writing was provided by Novartis Pharma AG.

Conflict of interest. Armando GonzalezRuiz received fees from Novartis, Pfizer, Cubist, and Gilead for staff training, being member of Advisory Boards, and being member of speakers panels. He also received support to attend scientific conferences including accommodation and travel payments, and a Novartis grant to support his research. Panayiotis Gargalianos-Kakolyris received fees from Novartis for speaking. Artur Timerman has nothing to disclose. Jayanta Sarma received support to attend one scientific conference including accommodation and travel payments and a Novartis grant to support his research. Víctor José González Ramallo received fees for being member of speakers panels and support to attend scientific conferences including accommodation and travel payments from Merck and Novartis. Kamel Bouylout is an employee of Novartis Pharma AG. Uwe Trostmann is a former employee of Novartis Pharma AG. Rashidkhan Pathan is an employee of Novartis Healthcare Pvt. Ltd. Kamal Hamed is an employee of Novartis Pharmaceuticals Corporation. 
Compliance with ethics guidelines. The protocol was approved by the health authority and the Institutional Review Board (IRB) or Ethics Committee (EC) in each country and written informed consent was obtained according to the requirements of the IRB or EC and/or the local data privacy regulations.

Open Access. This article is distributed under the terms of the Creative Commons Attribution Noncommercial License which permits any noncommercial use, distribution, and reproduction in any medium, provided the original author(s) and the source are credited.

\section{REFERENCES}

1. Steenbergen JN, Alder J, Thorne GM, Tally FP. Daptomycin: a lipopeptide antibiotic for the treatment of serious Gram-positive infections. J Antimicrob Chemother. 2005;55(3):283-8.

2. Shen HN, Lu CL. Skin and soft tissue infections in hospitalized and critically ill patients: a nationwide population-based study. BMC Infect Dis. 2010;10:151.

3. David MZ, Daum RS. Community-associated methicillin-resistant Staphylococcus aureus: epidemiology and clinical consequences of an emerging epidemic. Clin Microbiol Rev. 2010;23(3):616-87.

4. Dryden MS. Complicated skin and soft tissue infection. J Antimicrob Chemother. 2010;65 Suppl 3:iii35-44.

5. European Centre for Disease Prevention and Control. Antimicrobial resistance surveillance in Europe 2012. Annual Report of the European Antimicrobial Resistance Surveillance Network (EARS-Net). Stockholm: ECDC; 2013.

6. Deresinski S. Counterpoint: vancomycin and Staphylococcus aureus-an antibiotic enters obsolescence. Clin Infect Diseases. 2007;44(12):1543-8.

7. Rivera AM, Boucher HW. Current concepts in antimicrobial therapy against select gram-positive organisms: methicillin-resistant Staphylococcus aureus, penicillin-resistant pneumococci, and vancomycin-resistant enterococci. Mayo Clin Proc. 2011;86(12):1230-43.

8. Holland TL, Fowler VG Jr. Vancomycin minimum inhibitory concentration and outcome in patients with Staphylococcus aureus bacteremia: pearl or pellet? J Infect Dis. 2011;204(3):329-31.

9. Holmes NE, Turnidge JD, Munckhof WJ, et al. Antibiotic choice may not explain poorer outcomes in patients with Staphylococcus aureus bacteremia and high vancomycin minimum inhibitory concentrations. J Infect Dis. 2011;204(3):340-7.

10. Choi EY, Huh JW, Lim CM, et al. Relationship between the MIC of vancomycin and clinical outcome in patients with MRSA nosocomial pneumonia. Intensive Care Med. 2011;37(4):639-47.

11. Walsh TR, Howe RA. The prevalence and mechanisms of vancomycin resistance in Staphylococcus aureus. Annu Rev Microbiol. 2002;56:657-75.

12. Albrich WC, Angstwurm M, Bader L, Gartner R. Drug resistance in intensive care units. Infection. 1999;27(Suppl 2):S19-23.

13. Trick WE, Weinstein RA, DeMarais PL, et al. Colonization of skilled-care facility residents with antimicrobial-resistant pathogens. J Am Geriatr Soc. 2001;49(3):270-6.

14. Tiemersma EW, Bronzwaer SL, Lyytikainen O, et al. Methicillin-resistant Staphylococcus aureus in Europe, 1999-2002. Emerg Infect Dis. 2004;10(9):1627-34.

15. Novartis Europharm Ltd. Cubicin ${ }^{\circledR}$ (daptomycin) summary of product characteristics. 2012.

16. Arbeit RD, Maki D, Tally FP, Campanaro E, Eisenstein BI. The safety and efficacy of daptomycin for the treatment of complicated skin and skin-structure infections. Clin Infect Dis. 2004;38(12):1673-81.

17. Davis SL, McKinnon PS, Hall LM, et al. Daptomycin versus vancomycin for complicated skin and skin structure infections: clinical and economic outcomes. Pharmacotherapy. 2007;27(12):1611-8.

18. Gould IM, Miro JM, Rybak MJ. Daptomycin: the role of high-dose and combination therapy for Gram-positive infections. Int J Antimicrob Agents. 2013;42(3):202-10.

19. Kelesidis T. Pharmacological agents for the treatment of skin and soft tissue infections: what is the role for daptomycin? Clin Med Rev Ther. 2014;6:7-12. 
20. Gonzalez-Ruiz A, Beiras-Fernandez A, Lehmkuhl H, Seaton RA, Loeffler J, Chaves RL. Clinical experience with daptomycin in Europe: the first 2.5 years. J Antimicrob Chemother. 2011;66(4):912-9.

21. Fowler VG Jr, Boucher HW, Corey GR, et al. Daptomycin versus standard therapy for bacteremia and endocarditis caused by Staphylococcus aureus. N Engl J Med. 2006;355(7):653-65.

22. Seaton RA, Gonzalez-Ramallo VJ, Prisco V, et al. Daptomycin for outpatient parenteral antibiotic therapy: a European registry experience. Int J Antimicrob Agents. 2013;41(5):468-72.

23. Byren I, Rege S, Campanaro E, et al. Randomized controlled trial of the safety and efficacy of Daptomycin versus standard-of-care therapy for management of patients with osteomyelitis associated with prosthetic devices undergoing two-stage revision arthroplasty. Antimicrob Agents Chemother. 2012;56(11):5626-32.

24. Dohmen PM, Guleri A, Capone A, et al. Daptomycin for the treatment of infective endocarditis: results from a European registry. J Antimicrob Chemother. 2013;68(4):936-42.

25. Bubalo JS, Kullar R, Maziarz RT. A pilot study of the efficacy and safety of empiric daptomycin therapy in oncology patients with fever and severe neutropenia. Ther Adv Infect Dis. 2013;1(6):183-90.

26. Rybak JM, Barber KE, Rybak MJ. Current and prospective treatments for multidrug-resistant gram-positive infections. Expert Opin Pharmacother. 2013;14(14):1919-32.

27. Carugati M, Bayer AS, Miro JM, et al. High-dose daptomycin therapy for left-sided infective endocarditis: a prospective study from the international collaboration on endocarditis. Antimicrob Agents Chemother. 2013;57(12): 6213-22.

28. Legout L, D'Elia P, Sarraz-Bournet B, et al. Tolerability of high doses of daptomycin in the treatment of prosthetic vascular graft infection: a retrospective study. Infect Dis Ther. 2014;3(2):215-23.

29. Sakoulas G, Brown J, Lamp KC, Friedrich LV, Lindfield KC. Clinical outcomes of patients receiving daptomycin for the treatment of Staphylococcus aureus infections and assessment of clinical factors for daptomycin failure: a retrospective cohort study utilizing the Cubicin Outcomes Registry and Experience. Clin Ther. 2009;31(9):1936-45.
30. Seaton RA, Macconnachie AA. Experience with daptomycin in an infectious diseases service over 1 year: utility in an outpatient parenteral antibiotic programme. Int J Antimicrob Agents. 2008;31(5):492-7.

31. Stevens DL, Bisno AL, Chambers HF, et al. Practice guidelines for the diagnosis and management of skin and soft tissue infections: 2014 update by the Infectious Diseases Society of America. Clin Infect Dis. 2014;59(2):e10-52.

32. Gould FK, Denning DW, Elliott TS, et al. Guidelines for the diagnosis and antibiotic treatment of endocarditis in adults: a report of the Working Party of the British Society for Antimicrobial Chemotherapy. J Antimicrob Chemother. 2012;67(2):269-89.

33. Bosso JA, Nappi J, Rudisill C, et al. Relationship between vancomycin trough concentrations and nephrotoxicity: a prospective multicenter trial. Antimicrob Agents Chemother. 2011;55(12): 5475-9.

34. Lodise TP, Patel N, Lomaestro BM, Rodvold KA, Drusano GL. Relationship between initial vancomycin concentration-time profile and nephrotoxicity among hospitalized patients. Clin Infect Dis. 2009;49(4):507-14.

35. Rege S, Mohr J, Lamp KC, Yoon M, Lindfield KC. Safety of daptomycin in patients completing more than 14 days of therapy: results from the Cubicin(R) Outcomes Registry and experience. Int J Antimicrob Agents. 2013;41(5):421-5.

36. Ramsey TD, Lau TT, Ensom MH. Serotonergic and adrenergic drug interactions associated with linezolid: a critical review and practical management approach. Ann Pharmacother. 2013;47(4):543-60.

37. Lichterfeld M, Ferraro MJ, Davis BT. High-dose daptomycin for the treatment of endocarditis caused by Staphylococcus aureus with intermediate susceptibility to glycopeptides. Int J Antimicrob Agents. 2010;35(1):96.

38. Vidaillac C, Steed ME, Rybak MJ. Impact of dose deescalation and escalation on daptomycin's pharmacodynamics against clinical methicillinresistant Staphylococcus aureus isolates in an in vitro model. Antimicrob Agents Chemother. 2011;55(5):2160-5.

39. Gudiol F, Aguado JM, Pascual A, et al. Consensus document for the treatment of bacteremia and endocarditis caused by methicillin-resistent Staphylococcus aureus. Sociedad Espanola de Enfermedades Infecciosas y Microbiologia Clinica. Enferm Infecc Microbiol Clin. 2009;27(2):105-15. 
40. Casapao AM, Kullar R, Davis SL, et al. Multicenter study of high-dose daptomycin for treatment of enterococcal infections. Antimicrob Agents Chemother. 2013;57(9):4190-6.

41. Cheng CW, Hsu PC, Yang CC, et al. Influence of early daptomycin therapy on treatment outcome of meticillin-resistant Staphylococcus aureus bacteraemia with high vancomycin minimum inhibitory concentrations. Int $\mathrm{J}$ Antimicrob Agents. 2013;41(3):293-4.

42. Figueroa DA, Mangini E, Amodio-Groton M, et al. Safety of high-dose intravenous daptomycin treatment: three-year cumulative experience in a clinical program. Clin Infect Dis. 2009;49(2):177-80.

43. King EA, McCoy D, Desai S, Nyirenda T, Bicking K. Vancomycin-resistant enterococcal bacteraemia and daptomycin: are higher doses necessary? J Antimicrob Chemother. 2011;66(9):2112-8.

44. Kullar R, Casapao AM, Davis SL, et al. A multicentre evaluation of the effectiveness and safety of highdose daptomycin for the treatment of infective endocarditis. J Antimicrob Chemother. 2013;68(12):2921-6.
45. Kullar R, Davis SL, Levine DP, et al. High-dose daptomycin for treatment of complicated grampositive infections: a large, multicenter, retrospective study. Pharmacotherapy. 2011;31(6): 527-36.

46. Lai CC, Sheng WH, Wang JT, et al. Safety and efficacy of high-dose daptomycin as salvage therapy for severe gram-positive bacterial sepsis in hospitalized adult patients. BMC Infect Dis. 2013;13:66.

47. Murray KP, Zhao JJ, Davis SL, et al. Early use of daptomycin versus vancomycin for methicillinresistant Staphylococcus aureus bacteremia with vancomycin minimum inhibitory concentration $>1 \mathrm{mg} / \mathrm{L}$ : a matched cohort study. Clin Infect Dis. 2013;56(11):1562-9.

48. Bhavnani SM, Rubino CM, Ambrose PG, Drusano GL. Daptomycin exposure and the probability of elevations in the creatine phosphokinase level: data from a randomized trial of patients with bacteremia and endocarditis. Clin Infect Dis. 2010;50(12):1568-74. 